\title{
A Method for Quantifying Interaction Forces in Wearable Robots
}

\section{Conference Paper}

\section{Author(s):}

Georgarakis, Anna-Maria; Stämpfli, Rolf; Wolf, Peter (D); Riener, Robert; Duarte, Jaime E.

Publication date:

2018

Permanent link:

https://doi.org/10.3929/ethz-b-000307521

Rights / license:

In Copyright - Non-Commercial Use Permitted

Originally published in:

https://doi.org/10.1109/BIOROB.2018.8487701 


\title{
A Method for Quantifying Interaction Forces in Wearable Robots*
}

\author{
Anna-Maria Georgarakis ${ }^{1,2}$, Rolf Stämpfli ${ }^{3}$, Peter Wolf ${ }^{1}$, Robert Riener ${ }^{1,2}$ and Jaime E. Duarte ${ }^{1,2}$
}

\begin{abstract}
Immobility due to movement impairments causes many secondary conditions that are a threat to a person's health and quality of life. Wearable robotic mobility aids such as exoskeletons and exosuits are a promising technique to tackle immobility. These devices are attached to the human with cuffs. However, the physical interaction at the human-robot interface is not yet well understood. Misplacement and compression of soft tissue diminish the efficiency of the robot and the comfort for the human. We developed a measurement method that allows us to simultaneously measure cuff interaction forces in normal and tangential direction. The measurement setup was validated in a friction test bench. The test-retest reliability was evaluated in an isolated attachment cuff mounted on a human forearm. Force measurements were repeatable, with error ranges up to $28.7 \%$ or $7.8 \mathrm{~N}$ in normal, $28.7 \%$ or $2.3 \mathrm{~N}$ in tangential direction. Our method is the first approach that simultaneously measures normal and tangential forces at the physical interface of wearable robots. The test-retest reliability is within the range of methods that assess only normal forces.
\end{abstract}

\section{INTRODUCTION}

Movement impairments affect a person's quality of life. Next to the primary implication of immobility, movement impairments cause many secondary symptoms [1]. Classical mobility aids, such as wheelchairs, enable part of this population to regain mobility, but do not tackle the symptoms of continuous sitting, such as pressure sores, cardiovascular disease and digestive problems. More recently, exoskeletons have entered the market, which enable fully paralyzed patients to walk upright [2]. Similarly, textile based exosuits support patients with residual function. They support movements of the lower extremities when patients have become too weak to move against gravity [3][4]. Due to their low weight and high compliance, these devices are also eligible for wearable active assistance of the upper extremities.

To support movement, exosuits exert forces via pneumatics or tendon systems actuated by electrical motors. The soft compliant nature of exosuit devices is beneficial for user comfort and fit. It allows to integrate these systems seamlessly into the patients' lives. However, the compliance increases the complexity of the modeling and control of these systems [5]. Simulation of a non-rigid system acting on body shapes as diverse as mankind is impractical with the tools

*This research was supported by the Swiss National Science Foundation (SNSF) through the National Centre of Competence in Research (NCCR) Robotics

${ }^{1} \mathrm{~A}$ Georgarakis, $\mathrm{P}$ Wolf, $\mathrm{R}$ Riener and $\mathrm{J}$ Duarte are with the Sensory-Motor Systems (SMS) Lab, ETH Zurich, Switzerland marie.georgarakis@hest.ethz.ch

${ }^{2}$ A Georgarakis, R Riener and J Duarte are with the Spinal Cord Injury Center, Balgrist University Hospital, University of Zurich, Switzerland

${ }^{3} \mathrm{R}$ Stämpfli is with Empa, Swiss Federal Laboratories for Materials Science and Technology, Laboratory for Biomimetic Membranes and Textiles, St. Gallen, Switzerland rolf.staempfli@empa.ch at hand. The experimental analysis of wearable robots is, therefore, indispensable and an active field of research.

An important part of this analysis is the quantification of forces acting on the human. Wearable robots are attached to the human body with cuffs. Forces are exerted on the human by pulling or pushing on these cuffs. Depending on the line of action of the forces, both normal and tangential interactions can occur. The resulting forces are coupled due to friction and form fits. While some of these forces are wanted for mobilization, others are intrusive. The intrusive forces occur due to misalignment, deformation and displacement of the robot and the human. Intrusive forces influence the robot's performance, dynamics and controllability, and cause discomfort and harm for the human. The level of discomfort depends on the human's pain tolerance and skin condition, which vary greatly among individuals.

Human skin is more sensitive to shear than pressure. Next to discomfort, excessive shear causes severe skin damage. Moreover, patients suffering from sensory deficits may not be able to identify excessive skin shear. It is therefore critical to know the interaction forces occurring at the human-robot interface, in particular the tangential forces resulting in shear. Several groups have tried to quantify interaction pressures or forces in normal direction in exoskeletons [6][7][8][9] and exosuits [10] before. However, to date, no existing analysis was identified that quantified both forces in normal and tangential direction.

Further, as none of the above mentioned analyses evaluated the reliability of their method, generalization of their results is questionable. Four out of five of these analyses used force resistive sensors to measure pressure. The error ranges of such sensors expressed in terms of the coefficient of variation $C V$ has been reported to be up to $55 \%$ for average and up to $91 \%$ for peak pressure [11]. Additionally, the interaction between human skin and the robot cuffs depends on many parameters. Such dependencies are the cuff contact pressure, skin elasticity and humidity and changes in the muscle volume and compliance due to contraction [12][13].

The aim of our research is to quantify the interaction forces at the interface of a wearable robot and a human during use. Therefore, we developed a new measurement method that simultaneously measures forces in normal and tangential direction. Before testing on humans, we validated the setup in a test bench. To quantify the test-retest reliability, we performed repeated measurements on a human forearm. Here, reliability refers to the degree to which repeated measurements vary for an individual [14]. 


\section{METHODS}

The functioning principle of exosuits relies on force transfer via tendons or pneumatic actuation. We developed a measurement method that allows for quantification of interaction forces at the physical human-robot interface. Before integrating the measurement setup in an exosuit, the setup and its reliability were assessed in controlled test environments.

\section{A. Requirements}

A comprehensive analysis of the human-robot interaction forces requires the measurement of forces in normal and tangential directions. During short term load, comfortable stresses on the forearm range from 14 to $51.1 \mathrm{kPa}$ in normal and from 2.5 to $24 . \mathrm{kPa}$ in shear direction [16]. When permanent pressures above $4.26 \mathrm{kPa}$ are applied, the skin sustains structural damage [15]. Reoccurring loads during actuation should be at the lower boundary of these ranges.

The measurement system should be small enough to be integrated in the cuffs of wearable robots without profoundly changing the interface. From experience, the box size of the sensor setup should not exceed $100 \mathrm{~mm} \times 50 \mathrm{~mm} \times 20 \mathrm{~mm}$. Similarly, the weight should not exceed that of the actual cuff, which is about $0.2 \mathrm{~kg}$. To avoid pressure and force peaks, the transition between cuff and sensor system should be as smooth as possible.

\section{B. Measurement Setup}

Forces were only measured in part of the cuff. The sensor area needed to have a defined size and direction to extrapolate the pressures and stresses to the overall cuff area. The selected force sensor had a flat measurement surface and measured force in three dimensions (OMD-20-FE-200N, Optoforce, Budapest, Hungary) - one orthogonal to the plane and two in the plane of interaction. The sensor was embedded in a rigid casing to define the position in space the sensor had with respect to the interaction surface. The measurement method of the sensor relied on deformation due to exertion. The nominal bulk modulus of the sensor was approximately $K_{n}=0.167 \mathrm{GPa}$ in normal and $K_{t}=0.1 \mathrm{GPa}$ in tangential direction. Therefore, a substrate (Multifoam, $5 \mathrm{~mm}$ thickness) was added on the casing whose bulk modulus was determined to be approximately $K_{s}=0.002 \mathrm{GPa}<$ $K_{t} \wedge K_{n}$. Thereby, the deformation of the substrate did not cause a saturation of the sensor measurements. The substrate further aligned the sensor with the measurement plane in case of slight misalignment. After first tests, the decision was made to sandwich the substrate between two $1 \mathrm{~mm}$ aluminum plates to enhance the sensor surface and, therefore, force transmission. Therewithin, sensor readings were greatly improved at cost of a higher variability.

\section{Method Validation}

For the purpose of method validation, the setup was integrated into a test bench at Empa, St. Gallen, Switzerland (see Figure 1, left). The test bench was built to determine the friction coefficient of textiles against human skin. A

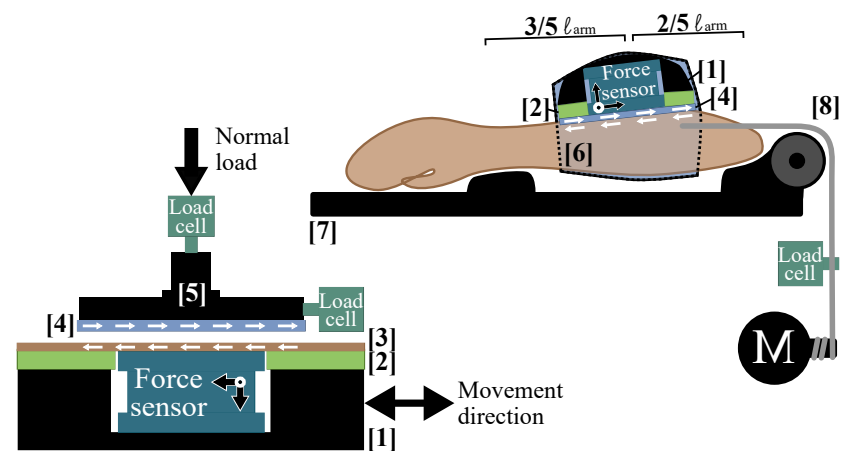

Fig. 1. Left: Friction measurement test bench. Tangential forces are displayed as white arrows. The Cartesian reference frame of the force sensor is indicated on the sensor in black. Right: Cuff measurement setup. A motor pulls on a tendon which is routed over pulleys and attached to a cuff. [1] Rigid Casing, [2] Substrate, [3] Skin substitute, [4] Textile, [5] Stamp, [6] Cuff, [7] Table with arm rests, [8] Pulley.

detailed description of the test bench can be found in [12]. The load cell measurements from the friction test bench were used as the ground truth for the sensor measurements. The textile used was a standard black bike jersey with a dynamic skin friction coefficient of 0.27 on dry and 0.41 on wet skin. The counterpart was a skin substitute (Lorica Soft, Ecolorica, Sri, Torino, Italy). Prior to any tests, the textiles were acclimatized for 24 hours in the test room at $20^{\circ} \mathrm{C}, 65 \%$ relative humidity. Two load cases were tested, $10 \mathrm{~N}$ and $20 \mathrm{~N}$ normal load. The interaction surface was $2500 \mathrm{~mm}^{2}$. To account for sweating, we tested dry and wet conditions ( $200 \mu \mathrm{l}$ of distilled water). The sledge of the test bench performed 730 cycles of which 700 were recorded as soon as the system was steady. The movement frequency was $1.25 \mathrm{~Hz}$, path length was $20 \mathrm{~mm}$. This results in a total of 4 measurements of 700 cycles. The sampling frequency of the force sensor was set to $100 \mathrm{~Hz}$. Due to technical difficulties in the data transmission, all measurements showed loss of single data points $(12.2 \% \ldots 28.0 \%$ per measurement). As sampling times were preserved, data could be uniquely identified. The sampling frequency of the load cells was set to $125 \mathrm{~Hz}$.

\section{Gain Calculation}

All data processing was done in python 3.6 using the numpy and scipy packages. A transfer gain was determined to calibrate the sensor measurement against the load cell measurements. Both force sensor and load cell data were filtered with a zero delay $2^{\text {nd }}$-order Butterworth filter. The cutoff frequency was set to $15 \mathrm{~Hz}$. Plotting the force values against each other revealed an approximately linear relationship between the data series. The non-linear least squares method curve_fit() was used to fit the data to the following polynomial:

$$
y_{l c}=K \cdot x_{f s}
$$

Data was fit both individually, for each of the four measurements $j$ to obtain the gains $K_{n \mid t}^{j}$, and to the whole data set to obtain the lumped gains $K_{n}$ for normal and $K_{t}$ for tangential forces. 


\section{E. Reliability Measurement Setup}

The reliability measurements were performed on one participant (Figure 1, right and Figure 2, right). A motor pulled on an isolated attachment cuff which was mounted on a human forearm. The forearm is an easily accessible limb segment. Due to its conic shape, it represents a suitable anchoring point for wearable robots. The force sensor setup was integrated in the cuff. The interaction surface was matched in size to the one used in the validation measurements. Both the sensor setup and the cuff were seamlessly covered in the same bike jersey as used in Section II-C. The force sensor data was recorded at $100 \mathrm{~Hz}$. The motor pulled on the cuff from both sides of the arm. A load cell (LSB200, Futek, CA, USA) was used to measure the tensile force of the motor and to control the force set points. Load cell data were recorded at $100 \mathrm{~Hz}$.

\section{F. Reliability Measurement Protocol}

The measurements were performed twice on the right forearm of a female participant aged 26 years. Before the measurement, the participant was seated in the measurement room for $20 \mathrm{~min}$ at $23^{\circ} \mathrm{C}, 40 \%$ relative humidity to acclimatize the skin. The participant did not apply cosmetics that day. She did not show any irritation or skin disease on the measurement site. The participant completed two consecutive measurement sessions on one day (Figure 2). The participant's skin was not expected to change from one session to the next. Each session was structured in exactly the same way, consisting of two blocks. In each block, six sub-blocks with different force profiles were presented, each repeated 30 times consecutively. Between each subblock, the participant had a $30 \mathrm{~s}$ break. During this time, the participant was encouraged to move their fingers to ensure blood circulation. The measurement setup was unand re-mounted before each block. The force sensors were calibrated before each mounting. To determine skin moisture levels, the electrical capacitance of the corneum stratum of the forearm was measured with a corneometer (Corneometer CM 825, Khazaka Electronic, Cologne, Germany). The humidity measurements were performed at a defined position on the bottom side of the forearm.

To ensure safety, pulling forces were within the range of comfortable shear stresses. Considering the surface of the testing cuff $\left(24116.5 \mathrm{~mm}^{2}\right)$, the maximum tensile force was set to $80 \mathrm{~N}$. Thus, resulting maximum stresses of $3.32 \mathrm{kPa}$ occurred, which were expected to have a normal and shear component. Additionally, a tensile force of $40 \mathrm{~N}$ was tested. Force profiles were chosen as to simulate two load conditions: slow, sinusoidal profiles as expected during continuous arm movements; and fast, impulsive profiles as expected during power transmission, e.g. when grasping an object. To control for the effect of muscle contractions, the participant was asked to either relax or contract the forearm muscles by grasping a handle. The participant was instructed to contract their muscles as steadily as possible. In the protocol, slow and fast profiles alternated. Every two profiles had the same maximum tensile force and grasp position.

\section{G. Data processing}

All data were filtered offline with a zero delay $2^{\text {nd }}$-order Butterworth filter with cutoff frequency set to $15 \mathrm{~Hz}$. For analysis, data were sliced into cycle repetitions and labeled chronologically. The first five repetitions were discarded due to shifts occurring predominantly in this period. Force sensor measurements were converted using verified conversion factors from the manufacturer. Tangential forces were combined using the Pythagorean theorem. Force measurements, $F_{n}$ in normal and $F_{t}$ in tangential direction, were scaled and shifted to compensate for the following effects: initial cuff attachment forces $F_{0 n}$ and $F_{0 t}$; the gains determined in the validation measurements $G_{n}$ and $G_{t}$; sensor and cuff area $A_{s}$ and $A_{c}$; motor tensile forces as determined by the load cell measurement $F_{l}$ scaled by the lumped transmission efficiency $\eta=0.7$. This transformation ensured that repeated measurements from different blocks are comparable.

$$
\begin{gathered}
F_{N}=\left(F_{n}-F_{0 n}\right) \cdot G_{n} \cdot \eta \cdot F_{l} \\
F_{T}=\left(F_{t}-F_{0 t}\right) \cdot G_{t} \cdot \eta \cdot F_{l} \cdot \frac{F_{n}}{F_{n}+F_{p n}}
\end{gathered}
$$

The last term in the second equation was used to compensate for the increase in shear force due to different initial normal cuff pressures (Coulomb's law of friction).

\section{H. Statistics}

Bland-Altman's limits of agreements analysis was used to compare the force measurement with the load cell measurement in the friction test bench [14]. Cycle repetitions were matched based on their chronological appearance in the protocol. As outcome measure for the analysis, the difference between the peak-to-peak amplitudes $\alpha_{p p, S 1}$ and $\alpha_{p p, S 2}$ was used. This means that we only consider the absolute changes in force during application of tensile forces, but not the forces caused by shifts and attachment pressures. To compare the measurement in session one $S 1$ with the re-test in session two $S 2$, we again used Bland-Altman's limits of agreements analysis. The analysis sets the difference between two repeated measures

$$
\Delta_{S 1 \mid S 2}=\alpha_{p p, S 1}-\alpha_{p p, S 2}
$$

in relation to their average

$$
\beta_{S 1 \mid S 2}=\frac{1}{2}\left(\alpha_{p p, S 1}+\alpha_{p p, S 2}\right) .
$$

To analyze the error, the range in which one expects $95 \%$ of the values for $\Delta_{S 1 \mid S 2}$ was calculated. This was done in terms of the standard deviation $\sigma$ and the coefficient of variation $C V$ [14].

$$
C V=100 \cdot 1.96 \cdot \frac{\sigma\left(\Delta_{S 1 \mid S 2}\right)}{\mu\left(\beta_{S 1 \mid S 2}\right)}
$$

A Student's t-test was used to determine whether the two repeated measurements had a significant bias. (ttest_rel() function, Python 3.6 scipy package). If so, this is reported according to

$$
\text { bias }=\mu\left(\Delta_{S 1 \mid S 2}\right) .
$$



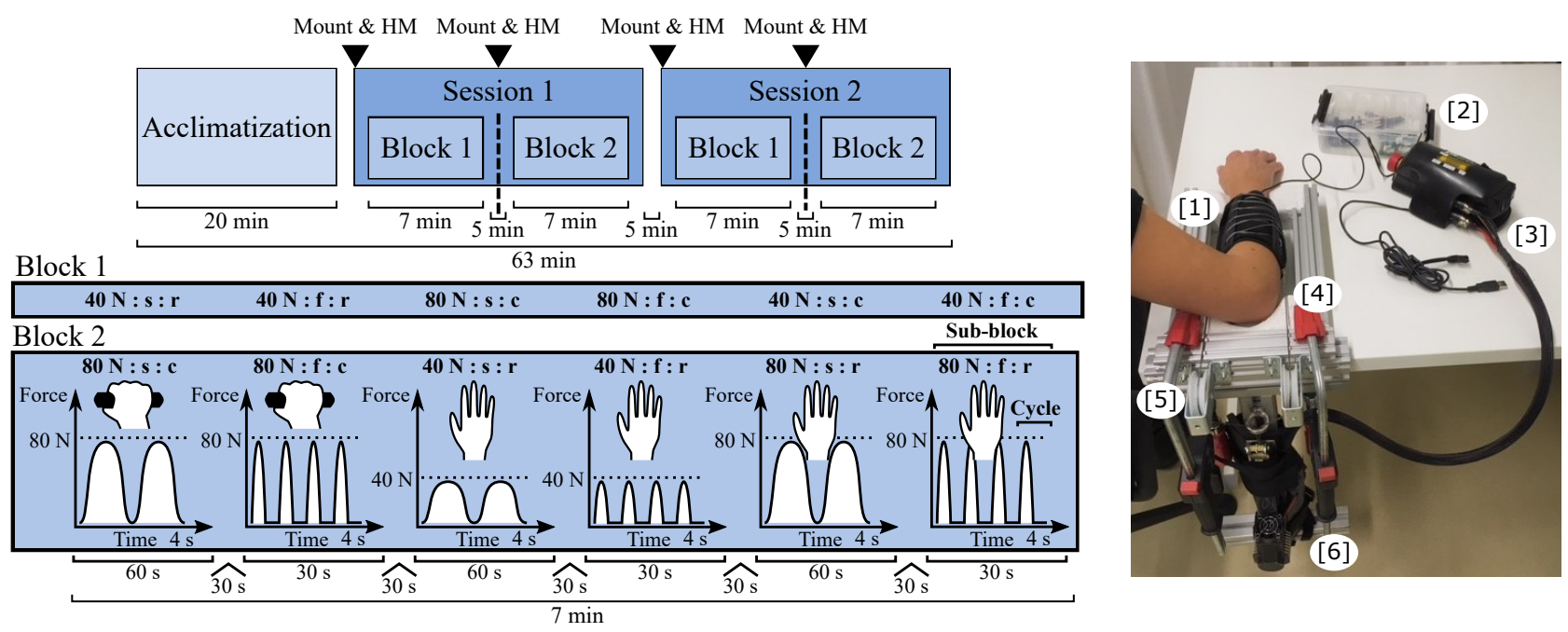

Fig. 2. Left: Measurement protocol. After an acclimatization period, the participant completed two sessions of two measurement blocks each. HM: skin humidity measurement. Sub-blocks are encoded in terms of maximum tensile force : slow (s) or fast (f) force profile : relaxed (r) or contracted (c) muscles. Right: Measurement setup. The cuff (1) is attached to the participant's forearm, which is rested on an arm rest (4). The motor (6) pulls on two tendons, which are routed over pulleys (5) and attached to the cuff. The measurement recording devices included the force sensor amplifier (2) and the control unit (3).

\section{RESULTS}

\section{A. Method Validation}

Force measurements were scaled by the individual gains $K_{i}^{j}$ and the lumped gains $K_{i}$, where $i=n, t ; j=1 \ldots 4$ (Table I and Figure 3). Lumped gains in normal direction were $K_{n}=3.49$, in tangential direction $K_{t}=1.34$.

\section{B. Reliability Measurements}

In the analysis, the data of sub-block 3 of block 2 (40 N : s : r) was excluded due to a protocol error concerning the hand position. The force profiles measured by the force sensor in normal and tangential direction coincide with the input profiles as determined by the load cell (Figure 4). In the normal direction, the means of the peak-to-peak amplitudes $\mu\left(\alpha_{p p, S 1}\right)$ and $\mu\left(\alpha_{p p, S 2}\right)$ range from $10.2 \mathrm{~N}$ to $40.4 \mathrm{~N}$ for a tensile force of $40 \mathrm{~N}$; from $29.4 \mathrm{~N}$ to $98.6 \mathrm{~N}$ for a tensile force of $80 \mathrm{~N}$. In tangential direction, they range from $0.3 \mathrm{~N}$ to $4.2 \mathrm{~N}$ for a tensile force of $40 \mathrm{~N}$; from $4.0 \mathrm{~N}$ to $26.8 \mathrm{~N}$ for a tensile force of $80 \mathrm{~N}$. Coefficients of variation $C V$ were a maximum of $28.7 \%$ (equivalent force range $\pm 3.7 \mathrm{~N}$ ) in normal and $28.7 \%$ (equivalent force range $\pm 0.3 \mathrm{~N}$ ) in tangential direction. Maximum force ranges were $\pm 7.8 \mathrm{~N}$ (equivalent $C V 16.1 \%$ ) in normal and $\pm 2.3 \mathrm{~N}$ (equivalent $C V 16.2 \%$ ) in tangential direction (Table II).

For the skin humidity measurements, all measurements were within the normal range as indicated by the manufacturer.

TABLE I

METHOD VALIDATION - Gains.

(NORMAL LOAD IN $[\mathrm{N}]$ : DRY $(d)$ OR WET $(w)$ MEASUREMENT)

\begin{tabular}{|c||c|c|c|c|c|}
\hline$j$ & $10 \mathrm{~N}: d$ & $10 \mathrm{~N}: w$ & $20 \mathrm{~N}: d$ & $20 \mathrm{~N}: w$ & lumped \\
\hline \hline$K_{n}^{j}$ & 3.01 & 3.11 & -3.37 & 3.89 & 3.49 \\
\hline$K_{t}^{j}$ & 1.53 & 1.45 & 1.31 & 1.31 & 1.34 \\
\hline
\end{tabular}

\section{DISCUSSION}

\section{A. Validation measurements}

The validation in the friction test bench showed consistent repetitions of force values during each measurement. Due to the compliance of the setup introduced by the sensor and the substrate, modeling of the force transmission was not straightforward. It was, therefore, decided to use these validation measurements to find a transfer gain between the baseline measurements and the measurements with the force sensor. The gains $K_{n / t}^{j}$ showed some deviations between the measurements $j$. Changing the normal load had a larger effect than increasing the humidity, which increased the friction coefficient. The third measurement done with a $20 \mathrm{~N}$ normal load on a dry textile showed considerable offsets in normal direction, presumably due to an error in the amplifier. However, the remaining three measurements improved their similarity to the baseline when scaled with the lumped gains $K_{n}$ and $K_{t}$. Due to compliant coupling in the setup, the force sensor slightly tilted during the measurements. The normal force measurements, therefore, reflect the movement of the test bench sledge, which are not present in the baseline. As the aim was to find a linear gain, these alternations in the forces were amplified by the gain. The nonlinear leastsquares method fitted the mean of the force sensor data to the baseline. However, the mean of the data reflects the normal force components and the alternations reflect portions of the tangential force. Therefore, it was decided not to correct for this circumstance.

\section{B. Test-retest measurements}

To estimate the reliability of the force measurements in a wearable robot, repeated measurements on an isolated attachment cuff were conducted. The cuff was mounted on a human forearm. The participant did not report any discomfort for the course of the measurements. The outcome measure 

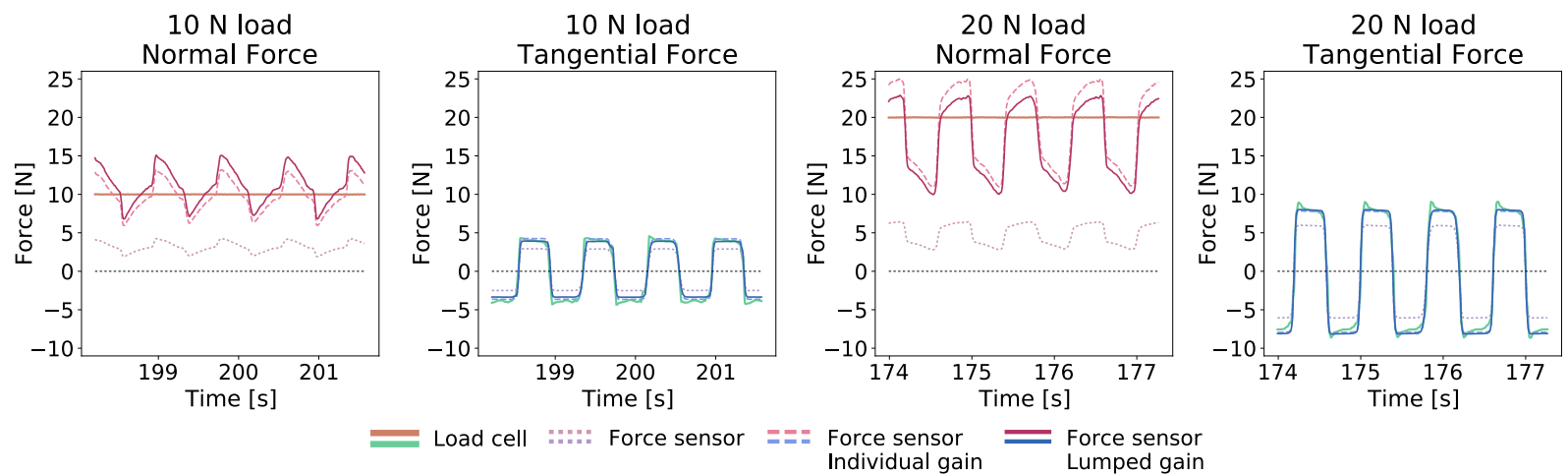

Fig. 3. Method validation results. Baseline values as determined by the load cells (bold lines); unscaled force measurements (dotted lines); force measurements scaled by individual gain fitted to respective measurement (dashed lines); force measurements scaled by lumped gain fitted to the overall data set (solid lines).

was the difference between the peak-to-peak amplitudes $\alpha_{p p}$ of the cyclic data for each of two measurement sessions, both in normal and tangential direction. Within one session, measurements showed good repeatability over 25 force profile cycles. When comparing the same measurements from different sessions, the resulting force profiles looked very similar, but showed considerable bias. The bias was diminished after normalization and scaling, but not completely, see Figure 4. Even though efforts were taken to mount the cuff equally before each block, positions and attachment pressures deviated. These variations are assumed to be the main reasons for the biases, an assumption that was affirmed by the results from the peak-to-peak analysis. However, further measurements and more profound modeling have to be done. In many cases, the peak-to-peak forces of one cycle in normal and tangential direction do not add up to the total force input from the motor (Table II). One reason for the mismatch may be the dynamic deformation of the compliant tissues, which introduced damping and delays to the system. Another reason could be the arm rest, which may have restricted the cuff during measurements.

A limits of agreement analysis of the means of peak-topeak amplitudes per sub-block was performed (Figure 4, [4] and [5]). In there, random errors increase with increasing systematic errors (biases), a sign for heterostadicity in the data. Data from block 1 appears to be a lot more congruent than in block 2. An explanation could be different cuff positions and shifts in block 2, and more similar positions in block 1. However, as cuff position was not measured during the measurements, this hypothesis could not be tested.

Due to Coulomb's law of friction, tangential forces are expected to scale proportionally with normal forces. Conversely, normal forces are expected to scale independently of tangential forces. However, in some cases higher normal forces led to lower tangential forces when comparing measurements between sessions. One reason could be lower initial shear stresses due to the cuff position. Another reason could be the nonlinear deformation of the tissue and the conic shape of the forearm. Due to these conditions, one cannot assume that the normal forces are uncoupled from the tangential forces, as in the friction test bench.
In block 2, the first two cycles were performed on contracted muscles with a maximum tensile force of $80 \mathrm{~N}$. Contracted muscles are less compliant. Decreased elasticity leads to increased slip instead of just elastic deformation of the cuff and forearm, which explains the increased forces in the following measurements on relaxed muscles.

\section{Comparison to previous work}

The presented method allows for simultaneous measurement of normal and tangential forces at the human-robot attachment cuffs of wearable robots. To date, no publication was identified that showed a comparable method. The error ranges obtained in the peak-to-peak analysis were lower than the ones Wettenschwiler et al. found for the Tekscan sensors [11]. Due to the differences in protocol, data acquisition and analysis, a direct comparison of these results must be done carefully. However, the determined error ranges lead to think that it is worthwhile to further pursue our method in future studies.

\section{Limitations}

Next to the points mentioned above, further limitations were identified in our setup, measurements and analysis. In the current analysis, data from one participant was considered. Hence, the data is dependent, which is a major limitation. Data from different participants is required to determine the inter-subject reliability of the presented method. So far, data acquisition from different sources was not synchronized, leading to errors due to time lags. The load cell in the reliability measurement setup was mounted in series to the tendons at the motor. Therefore, it measured the pulling force of the motor and not on the cuff. Due to friction and other losses, these pulling forces deviated. At the point of writing, the error introduced by these effects could not be estimated.

\section{CONCLUSION}

The presented method is the first that allows for simultaneous measurement of normal and tangential forces in the attachment cuffs of wearable robots. The reliability analysis revealed errors that lie within the ranges of methods analyzing only normal forces. The setup can be used for detailed analyses of the physical interfaces of wearable robots. 

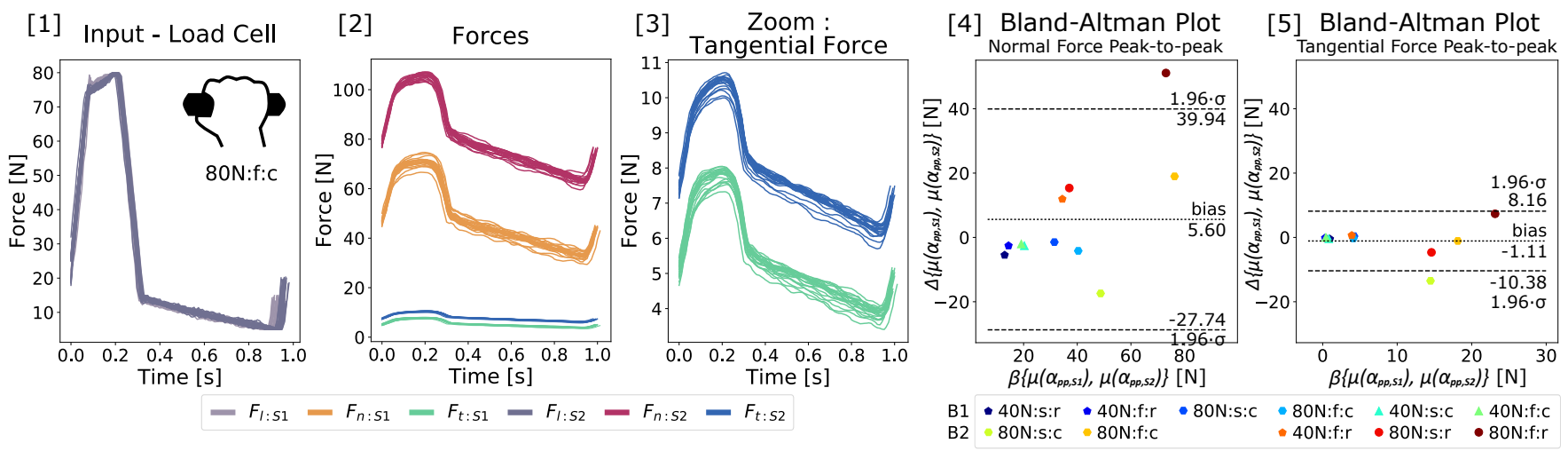

Fig. 4. [1,2,3]: Thirty fast, impulsive force profiles with a maximum tensile force of $80 \mathrm{~N}$ were presented (Block 1, Sub-block 4). Muscles were contracted during the measurement. Normal forces are about ten times larger than tangential forces. Even though a considerable bias is present, force profiles are congruent. [4,5]: Bland-Altman plots of the means of normal and tangential peak-to-peak amplitudes per sub-block. Whereas the bias is a measure for the systematic error in the data (bias), the standard deviation represents the random errors $(1.96 \cdot \sigma)$. For legend decoding, see Figure 2.

TABLE II

PEAK-TO-PEAK ANALYSIS

Sub-Blocks. MAXimum tensile Force : SLOW $(s)$ OR FASt $(f)$ FORCE PROFILE : RELAXed $(r)$ OR CONTRACTED $(c)$ MUSCLES $\mathrm{BIAS}=\mu\left(\Delta_{S 1 \mid S 2}\right) \quad\left|\quad \mathrm{CV}=100 \cdot 1.96 \cdot \frac{\sigma\left(\Delta_{S 1 \mid S 2}\right)}{\mu\left(\beta_{S 1 \mid S 2}\right)} \quad\right| \quad \mathrm{RANGE}=1.96 \cdot \sigma\left(\Delta_{S 1 \mid S 2}\right)$

\begin{tabular}{|c|c|c|c|c|c|c|c|c|c|c|c|}
\hline & \multirow[b]{2}{*}{ Sub-block } & \multicolumn{5}{|c|}{ Normal } & \multicolumn{5}{|c|}{ Tangential } \\
\hline & & $\begin{array}{c}\mu\left(\alpha_{p p, S 1}\right) \\
{[N]}\end{array}$ & $\begin{array}{c}\mu\left(\alpha_{p p, S 2}\right) \\
{[N]}\end{array}$ & $\begin{array}{l}\text { bias } \\
{[N]}\end{array}$ & $\begin{array}{l}\mathrm{CV} \\
{[\%]}\end{array}$ & $\begin{array}{l}\text { range } \\
{[ \pm N]}\end{array}$ & $\begin{array}{c}\mu\left(\alpha_{p p, S 1}\right) \\
{[N]}\end{array}$ & $\begin{array}{c}\mu\left(\alpha_{p p, S 2}\right) \\
{[N]}\end{array}$ & $\begin{array}{l}\text { bias } \\
{[N]} \\
\end{array}$ & $\begin{array}{l}\mathrm{CV} \\
{[\%]}\end{array}$ & $\begin{array}{l}\text { range } \\
{[ \pm N]}\end{array}$ \\
\hline \multirow{6}{*}{ Block 1} & $40 \mathrm{~N}: s: r$ & 10.2 & 15.7 & -5.5 & 28.7 & 3.7 & 0.7 & 1.2 & -0.5 & 28.7 & 0.3 \\
\hline & $40 \mathrm{~N}: f: r$ & 13.1 & 15.7 & -2.6 & 10.9 & 1.6 & 0.3 & 0.4 & -0.2 & 12.1 & 0.0 \\
\hline & $80 \mathrm{~N}: s: c$ & 30.7 & 32.2 & -1.5 & 13.5 & 4.3 & 4.4 & 4.0 & 0.4 & 11.7 & 0.5 \\
\hline & $80 \mathrm{~N}: f: c$ & 38.3 & 42.5 & -4.2 & 7.6 & 3.1 & 4.0 & 4.3 & -0.3 & 6.2 & 0.3 \\
\hline & $40 \mathrm{~N}: s: c$ & 19.0 & 21.6 & -2.6 & 18.3 & 3.7 & 0.6 & 1.0 & -0.4 & 20.3 & 0.2 \\
\hline & $40 \mathrm{~N}: f: c$ & 18.1 & 20.2 & -2.0 & 18.1 & 3.5 & 0.4 & 0.4 & 0.0 & 13.6 & 0.1 \\
\hline \multirow{5}{*}{ Block 2} & $80 \mathrm{~N}: s: c$ & 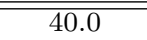 & $\overline{57.4}$ & $\overline{-17.4}$ & $\overline{16.1}$ & $\overline{7.8}$ & 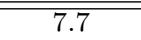 & $\overline{21.2}$ & $\overline{-13.5}$ & $\overline{16.2}$ & $\overline{2.3}$ \\
\hline & $80 \mathrm{~N}: f: c$ & 85.8 & 66.8 & 19.0 & 4.8 & 3.7 & 17.6 & 18.7 & -1.1 & 4.8 & 0.9 \\
\hline & $40 \mathrm{~N}: f: r$ & 40.4 & 28.5 & 11.9 & 5.6 & 1.9 & 4.2 & 3.6 & 0.6 & 6.7 & 0.3 \\
\hline & $80 \mathrm{~N}: s: r$ & 44.7 & 29.4 & 15.4 & 18.8 & 7.0 & 12.3 & 16.9 & -4.7 & 15.1 & 2.2 \\
\hline & $80 \mathrm{~N}: f: r$ & 98.6 & 47.5 & 51.1 & 5.9 & 4.3 & 26.8 & 19.5 & 7.3 & 6.0 & 1.4 \\
\hline
\end{tabular}

\section{ACKNOWLEDGMENT}

The authors would like to thank Patrizia Ballistreri and Laura Knezevic for their technical support.

\section{REFERENCES}

[1] E. G. Wilmot, C. L. Edwardson, F. A. Achana et al., Sedentary time in adults and the association with diabetes, cardiovascular disease and death: systematic review and meta-analysis. Diabetologia, vol. 55, pp. 2895-2905, 2012

[2] A. J. Young and D. P. Ferris, State of the Art and Future Directions for Lower Limb Robotic Exoskeletons. IEEE Transactions on Neural Systems and Rehabiliitation Engineering, vol. 25, pp. 171-182, 2017

[3] K. Schmidt, J. E. Duarte, M. Grimmer et al., The Myosuit: Bi-articular Anti-gravity Exosuit That Reduces Hip Extensor Activity in Sitting Transfers. Fontiers in Neurorobotics, vol. 11, pp. 1-16, 2017

[4] L. N. Awad, J. Bae, K. O'Donnel et al., Awad, Louis N., et al. A soft robotic exosuit improves walking in patients after stroke. Science translational medicine, vol. 9, 400, 2017

[5] M. B. Yandell, B. T. Quinlivan, D. Popov et al., Physical interface dynamics alter how robotic exosuits augment human movement: implications for optimizing wearable assistive devices. Journal of NeuroEngineering and Rehabilitation, vol. 14, pp 1-11, 2017

[6] S. De Rossi, N. Vitiello, T. Lenziet al., Sensing Pressure Distribution on a Lower-Limb Exoskeleton Physical Human-Machine Interface. Sensors, vol.11, pp.207-227, 2011

[7] M. Wilcox, A. Rathore, D. Morgado Ramirez et al., Muscular activity and physical interaction forces during lower limb exoskeleton use. Healthcare Technology Letters, vol. 3, pp. 273-279, 2016
[8] A. Rathore, M. Wilcox, D. Morgado Ramirez et al., Quantifying the human-robot interaction forces between a lower limb exoskeleton and healthy users. In Proceedings of the Annual International Conference of the IEEE Engineering in Medicine and Biology Society (EMBC) 2016, pp.586-589, 2016

[9] J. Tamez-Duque, R. Cobian-Ugalde, A. Kilicarslan et al., Real-Time Strap Pressure Sensor System for Powered Exoskeletons. Sensors, vol.15, pp.4550-4563, 2015

[10] B. Quinlivan, A. Asbeck, D. Wagner et al., Force Transfer Characterization of a Soft Exosuit for Gait Assistance. Volume 5A: 39th Mechanisms and Robotics Conference, 2015

[11] P. Wettenschwiler, R. Stämpfli, S. Lorenzetti et al., How reliable are pressure measurements with Tekscan sensors on the body surface of human subjects wearing load carriage systems?. International Journal of Industrial Ergonomics, vol. 49, pp. 60-67, 2015

[12] S. Derler, U. Schrade and L. Gerhardt, Tribology of human skin and mechanical skin equivalents in contact with textiles. Wear, vol. 263, pp. 1112-1116, Nov. 2006

[13] C. Hendriks and S. Franklin, Influence of Surface Roughness, Material and Climate Conditions on the Friction of Human Skin. Tribology Letters, vol. 37, pp. 361-373, 2010

[14] G. Atkinson and A. Nevill, Statistical Methods For Assessing Measurement Error (Reliability) in Variables Relevant to Sports Medicine. Sports Medicine, vol. 26, pp.217-238, 1998

[15] C. Giuglea, S. Marinescu, I. P. Florescu et al., Pressure sores: a constant problem for plegic patients and a permanent challenge for plastic surgery. Journal of medicine and life, vol. 3, pp 149-153, 2010

[16] A. Manorama, S. Baek, J. Vorro et al., Blood perfusion and transcutaneous oxygen level characterizations in human skin with changes in normal and shear loads? Implications for pressure ulcer formation. Clinical Biomechanics, vol. 25, pp. 823-828, June 2010 